\title{
25 Research Soure

\section{Pharmacokinetics and Bioequivalence of Sitagliptin Phosphate/Metformin Hydrochloride Tablets in Healthy Chinese Subjects: A Randomized, Open, Crossover Study}

\section{Ping SHI}

The Affiliated Hospital of Qingdao University

\section{Xin LIU}

Qingdao University

\section{Ting LI}

The Affiliated Hospital of Qingdao University

\section{Fei-fei SUN}

The Affiliated Hospital of Qingdao University

Yan-ping LIU

The Affiliated Hospital of Qingdao University

\section{Shu-qin LIU}

The Affiliated Hospital of Qingdao University

\section{Xiao-meng GAO}

The Affiliated Hospital of Qingdao University

\section{Ya-ping MA}

The Affiliated Hospital of Qingdao University

\section{Yao FU}

The Affiliated Hospital of Qingdao University

Yu CAO ( $\nabla$ caoyu1767@163.com )

The Affiliated Hospital of Qingdao University

\section{Research Article}

Keywords: Sitagliptin Phosphate/metformin Hydrochloride Tablets, pharmacokinetics, bioequivalence, healthy Chinese subjects

Posted Date: September 14th, 2021

DOl: https://doi.org/10.21203/rs.3.rs-757296/v1 
License: (c) (i) This work is licensed under a Creative Commons Attribution 4.0 International License. Read Full License

Version of Record: A version of this preprint was published at Drugs in R\&D on November 30th, 2021. See the published version at https://doi.org/10.1007/s40268-021-00371-2. 


\section{Abstract}

Objective To evaluate the pharmacokinetics and bioequivalence between Sitagliptin Phosphate/metformin Hydrochloride Tablets (test formulation) at a single dose of 50mg/850mg and Sitagliptin Phosphate/metformin Hydrochloride Tablets (reference formulation, JANUMET ${ }^{\circledR}$ ) at a single dose of $50 \mathrm{mg} / 850 \mathrm{mg}$ in healthy Chinese subjects.

Methods The study was designed as a randomized, open-lable, two-period double-crossover trial. A total of 24 volunteers under fasting condition and 24 subjects under fed condition were given a single oral dose of $50 \mathrm{mg} / 850 \mathrm{mg}$ Sitagliptin Phosphate/metformin Hydrochloride Tablets of test and reference, respectively. Liquid chromatography Tandem mass spectrometry (LC-MS/MS) method was used to determine the concentrations of sitagliptin and metformin in the plasma of subjects. The pharmacokinetic parameters were calculated by WinNonlin 7.0 program and the bioequivalence was evaluated by SAS 9 . 4program.

Results The $90 \%$ confidential interval (Cl) of geometric mean ratio under fasting condition for $C_{\text {max }}, A C_{0}$ ${ }_{t}$ and $\mathrm{AUC}_{0-\infty}$ of sitagliptin between the test group and the reference group were $101.70 \%-120.62 \%$, 99.81\%-105.61\%, 100.27\%-106.12\% and metformin were 90.39\%-111.48\%, 94.76\%-109.12\%, 95.76\%-110.38\%. The $90 \% \mathrm{Cl}$ of geometric mean ratio under fed condition for $\mathrm{C}_{\max }, \mathrm{AUC}_{0-\mathrm{t}}$ and $\mathrm{AUC}_{0-\infty}$ between the test group and the reference group were $102.12 \%-117.31 \%, 100.80 \%-107.81 \%$, 100.82\%-107.78\% and metformin were $95.53 \%-105.22 \%, 92.76 \%-103.07 \%, 93.40 \%-104.14 \%$. Both were generally well tolerated.

Conclusion The two formulations of Sitagliptin Phosphate/metformin Hydrochloride Tablets were bioequivalent under fasting and fed condition in healthy Chinese subjects.

Trial registration Clinical Trial Registry (trial ID: NCT04877106).

\section{Introduction}

The number of diabetic patients worldwide has reached 366 million in 2011, the number of diabetic patients will reach 552 million by 2030, according to official statistics from the International Diabetes Federation (IDF). [1] Type 2 diabetes mellitus (T2DM) accounts for 93.7 percent of the total which is currently the fastest growing type of patients and the most studied type. [2] Most of the serious complications of diabetes are macrovascular and microvascular diseases caused by accelerated atherosclerosis. The cardiovascular morbidity is $2-4$ times higher in people with type 2 diabetes than in people without diabetes. [3]

Metformin is the initial treatment recommended in almost all guidelines for the initial treatment of type 2 diabetes and is widely used in the clinic. [4] However, metformin alone often fails to meet the basic requirements for glycemic control. [5] Therefore, it is necessary to use oral hypoglycemic drugs in combination to effectively manage the blood glucose of diabetic patients. Common combination 
hypoglycemic drugs include Sulfonylureas, Acarbose, DPP-4 inhibitors, GLP1 receptor agonists and SGLT2 inhibitors.

Sitagliptin showed a strong and highly selective inhibition of DPP-4. [6] DPP-4 inhibitors have a wide range of therapeutic applications. The risk of hypoglycemia is low when used alone or in combination with other drugs that do not cause hypoglycemia. And DPP-4 inhibitors did not cause weight gain. [4] Sitagliptin improves glycemic control by inhibiting DPP4 inactivation of GLP-1 and glucose-dependent GIP. Inhibition of DPP-4 increases and prolongs the activity level of incretin, leading to a glucosedependent increase in insulin release and a decrease in glucagon. [7]

The combination of sitagliptin and metformin has been shown to have a significant effect on blood glucose control in patients with type 2 diabetes, and is well tolerated with a low risk of hypoglycemia. [8] Sitagliptin Phosphate/metformin Hydrochloride Tablets are more effective than any single ingredient in the blood sugar control of type 2 diabetic patients. [9] Currently, Sitagliptin Phosphate/metformin Hydrochloride Tablets (JANUMET ${ }^{\circledR}$ ) have been approved for the treatment of type 2 diabetes. The purpose of the study is to study the pharmacokinetics of the test and reference formulations (sitagliptin $50 \mathrm{mg}$ and metformin $850 \mathrm{mg}$ ) of sitagliptin and metformin tablets, and to evaluate the pharmacokinetics and bioequivalence.

\section{Methods}

\section{Compliance with Ethics Guidelines}

This clinical study protocol was approved by the Medical Ethics Committee at the Affiliated Hospital of Qingdao University and the trial was registered with the Clinical Trial Registry (trial ID: NCT04877106). All procedures were performed in compliance with the Declaration of Helsinki [10] as well as the International Conference on Harmonization Guideline for Good Clinical Practice [11]. All volunteers provided written informed consent before being screened for eligibility.

\section{Study Population}

Eligible study participants included healthy, male and female subjects aged $18-45$ years with a body mass index $18-28 \mathrm{~kg} / \mathrm{m}^{2}$. Subjects (including male subjects) are willing to have no family planning and voluntarily use effective contraceptive measures for the next 6 months. Subjects sign the informed consent before the trial, and have a full understanding of the content, process and possible adverse reactions. The exclusion criteria included as follows: Being allergy to the study medications, smoking, alcohol abuse, and participation in another clinical trial within 3 months. pregnant or nursing females; use of prescription or non-prescription drugs (including herbal remedies).

\section{Study Design}

An open-label, randomized, single-dose, two-period, crossover study was conducted at the Phase I Clinical Research Center of the Affiliated Hospital of Qingdao University. Randomization was conducted by the 
investigators in accordance with a randomization schedule, created using the SAS 9.4 Proc Plan process by the statistical analysis unit for fasting and fed conditions, respectively.

Sitagliptin Phosphate/metformin Hydrochloride Tablets [50mg/850mg, batch no. M047893, manufactured by MSD Pharma (Singapore) Pte.Ltd] as reference formulations and Sitagliptin Phosphate/metformin Hydrochloride Tablets $(50 \mathrm{mg} / 850 \mathrm{mg}$, batch no. 161006 , manufactured by Tonghua Dongbao Pharmaceutical Co., Ltd.) as test formulations were used in this study. All study drugs were provided by the sponsor (Tonghua Dongbao Pharmaceutical Co., Ltd.) at no cost. All subjects were hospitalized in the phase I clinical research center on the day before dosing and placed on a uniform diet during hospitalization. Subjects in the fasting group (24 subjects) were asked to fast overnight $(10 \mathrm{~h}$ ) before administration, while the subjects in the fed group (24 subjects) needed to take an additional highfat, high-calorie standard meal 30 min prior to the administration. Test and reference formulations of Sitagliptin Phosphate/metformin Hydrochloride Tablets was swallowed with $240 \mathrm{ml}$ water at room temperature. Subjects were allocated to one of two groups randomly and equally with a 7-day washout interval between the two periods (Fig. 1).

Caffeine-containing beverages, food rich in xanthine, fruits that affect drug metabolism (e.g., grapefruit, dragon fruit, mango) and alcohol were prohibited from $48 \mathrm{~h}$ prior to dosing to the completion of blood sampling.

\section{Blood Sampling}

Blood samples ( $4 \mathrm{ml}$ ) were collected in vacuum tubes containing EDTA-K 2 at pre-dosing $(0 \mathrm{~h})$ and $0.5,1$, $1.5,2,2.5,3,3.5,4,5,6,8,10,12,16,24,32,38$ and $72 \mathrm{~h}$ after dosing in each study period. The samples were centrifuged at $3000 \mathrm{rpm}$ for $10 \mathrm{~min}$ at $2-8^{\circ} \mathrm{C}$ to separate the plasma. Samples were divided into two aliquots and stored at $-80^{\circ} \mathrm{C}$ until analysis.

\section{Pharmacokinetic Methods}

Plasma samples were determined at Junke Zhengyuan (Beijing) Pharmaceutical Research Co., Ltd., and analyzed by LC MS/MS methods, which were validated in terms of selectivity, accuracy, precision, recovery and stability. The LC-MS/MS system consisted of a Shimadzu UFLC 20-AD ultrafast liquid phase system (Shimadzu Corp., Kyoto, Japan) and API 4000 mass spectrometer (Ap- plied Biosystems, MDS Sciex). Metformin: mobile phase A consisted of $0.1 \%$ FA (by volume) containing $5 \mathrm{mMammonium}$ acetate and mobile phase B consisted of $0.1 \%$ FA (by volume) in ACN. Sitagliptin: mobile phase A consisted of $0.1 \%$ FA (by volume) in water and mobile phase B consisted of $0.1 \%$ FA (by volume) in ACN. API 4000 was operated in the positive ion multiple reaction monitoring (MRM) mode using Turbo Spray. The lower limit of quantification (LLQ) for metformin the plasma assay was $3 \mathrm{ng} / \mathrm{mL}$ and the linear calibration range was $3-1500 \mathrm{ng} / \mathrm{mL}$. The overall precision of the metformin assay was $8.9 \%$ coefficient of variation (CV). The lower limit of quantification (LLQ) for sitagliptin the plasma assay was $1 \mathrm{ng} / \mathrm{mL}$ and the linear calibration range was $1-500 \mathrm{ng} / \mathrm{mL}$. The overall precision of the sitagliptin assay was $5.8 \%$ coefficient of variation (CV). 


\section{Safety and Tolerability Assessments}

Data from all study participants were included in safety and tolerability assessments. Evaluation of safety was accomplished through subject-reported adverse events, investigator observations and assessments, and prespecified study evaluations (laboratory safety tests, ECGs and vital sign measurements). Safety and tolerability were evaluated by clinical review of all safety parameters.

\section{Statistical Analysis}

Analysis of variance (ANOVA) was performed on the logarithmically transformed values of the primary PK parameters $\left(\mathrm{C}_{\text {max }}, \mathrm{AUC}_{0-t}\right.$, and $\left.\mathrm{AUC}_{0-\infty}\right)$. The ratios of geometric means $(\mathrm{GM})$ of these primary PK parameters (test/reference) and their $90 \%$ confidence intervals (Cls) were calculated. The two preparations were considered bioequivalent if the $90 \%$ Cls of the ratios of GM of the primary PK parameters were within the predefined acceptance range of $80-125 \%$. The independent samples t-test was used for comparisons of logarithmically transformed primary PK parameters of the test formulation. All PK parameter analysis was calculated by WinNonlin 7.0, while other data processing and statistical analyses were performed by SAS 9.4.

\section{Results}

\section{Subject Disposition}

The overall study population enrolled 48 subjects (fasting group 24, fed group24). All of 24 subjects (14 males, 10 females) in the fasting group completed the study. The demographic details were as follows (mean \pm SD): age was $28.9 \pm 8.28$ years, height $166.17 \pm 7.299 \mathrm{~cm}$, weight $61.54 \pm 7.699 \mathrm{~kg}$ and body mass index (BMI) $22.25 \pm 1.789 \mathrm{~kg} / \mathrm{m}^{2}$. A total of 24 healthy subjects ( 15 males and 9 females) were enrolled in the fed group. Twenty-three subjects completed the two treatment periods while one subject took the initiative to drop out during the baseline before the second treatment period. The demographic details were as follows (mean \pm SD): age was $25.6 \pm 4.69$ years, height $168.27 \pm 10.140 \mathrm{~cm}$, weight $62.56 \pm 8.779$ $\mathrm{kg}$ and body mass index (BMI) $22.03 \pm 1.850 \mathrm{~kg} / \mathrm{m} 2$.

\section{Pharmacokinetics}

\section{Fasting group}

The plasma concentration-time profiles for sitagliptin and metformin under fasting conditions are shown respectively in Fig. 2 and Fig.3. Summaries of PK parameters for sitagliptin and metformin are presented in Table 1. ANOVA indicated a lack of period and sequence effects for $C_{\text {max }}, A C_{0-t}$, and $A U C_{0-\infty}$. There was a statistically significant difference between formulations in $C_{\max }, A \cup C_{0-t}$, and $A U C_{0-\infty}(p \leq 0.05)$. The $90 \%$ Cls for the geometric mean ratios of $\mathrm{C}_{\text {max }}, \mathrm{AUC}_{0-t}$, and $\mathrm{AUC}_{0-\infty}$ and the power of the test are presented in Table 1, and all of them were within the predefined equivalence limit of 0.80-1.25.

\section{Fed group}


The mean plasma concentration-time profiles for sitagliptin and metformin under fed conditions are shown respectively in Fig. 4 and Fig. 5 Summaries of PK parameters for sitagliptin and metformin are presented in Table 2. ANOVA indicated a lack of period and sequence effects for $C_{\text {max }}, A C_{0-t}$, and $A U C_{0-\infty}$. There was a statistically significant difference between formulations in $\mathrm{C}_{\text {max }}, \mathrm{AUC}_{0-\mathrm{t}}$, and $\mathrm{AUC}_{0-\infty}$ $(p \leq 0.05)$. The $90 \%$ Cls for the geometric mean ratios of $C_{\text {max }}, A U C_{0-t}$ and $A_{U-\infty}$ and the power of the test are presented in Table 2, and all of them were within the predefined equivalence limit of 0.80-1.25.

\section{Safety and Tolerability}

Both test and reference formulations were generally well tolerated during the entire study periods. There were no protocol violations or serious adverse events (SAEs) observed in the study under either fasting or fed conditions. Four volunteers experienced a total of $4 \mathrm{AEs}$ in fasting group and the fed group respectively. The AEs were listed in Table3 and Table4

\section{Discussion}

Type 2 diabetes mellitus (T2DM) is a complex metabolic disease with an increasing number of patients each year. [12] Sitagliptin is a dipeptidyl peptidase-4 inhibitor (DPP-4), a newer treatment for type 2 diabetes mellitus. [13] Metformin hydrochloride is a biguanide, widely viewed as foundation therapy for type 2 diabetes mellitus. [14] More and more researches suggest that combination tablets will be an increasingly common strategy for the treatment of type 2 diabetes.[8] Sitagliptin Phosphate/metformin Hydrochloride Tablets is a single-tablet, fixed-dose combination of the dipeptidyl peptidase- 4 inhibitor sitagliptin and the biguanide anti-hyperglycemic metformin which has become an important treatment for type 2 diabetes. [15] In this study, sitagliptin and metformin in plasma were quantitatively analyzed, and the pharmacokinetic characteristics and safety of domestic and original Sitagliptin Phosphate/metformin Hydrochloride Tablets were investigated, and the bioequivalence of the two preparations was evaluated. To provide multiple options for clinical combination regimens for type 2 diabetes.

Here, we assess the bioequivalence of Sitagliptin Phosphate/metformin Hydrochloride Tablets under fasting and fed conditions in healthy Chinese subjects following a single oral dose of $50 \mathrm{mg} / 850 \mathrm{mg}$ test (Tonghua Dongbao Pharmaceutical Co., Ltd.) and reference (MSD Pharma (Singapore) Pte.Ltd) formulations. The two medicinal products are bioequivalent when their $90 \% \mathrm{Cl}$ of the $\mathrm{AUC}_{0-t}, \mathrm{AUC}_{0-\infty}$ and Cmax of the reference preparation over the test preparation fall between the predetermined limits of $80 \sim$ $125 \%$.

According to our results, both formulations were well tolerated, and no SAEs was observed during the study. All reported AEs were of mild intensity, and no subjects withdrew from the study because of any AEs. The main adverse drug reactions were nausea, hyperuricemia, and hyperkalemia. Most of the adverse drug reactions in this trial are similar to those reported in the literature, indicating that the test and reference formulations have good safety and tolerability in Chinese healthy subjects. [16] The 
reference formulations specifications and previous literature have reported that food can decrease metformin absorption and slightly delay it absorption.[17] The main pharmacokinetic parameters of oral reference formulations metformin in fed group were significantly lower than those of in fasting group, $\mathrm{C}_{\text {max }}, \mathrm{AUC}_{0-\mathrm{t}}$ and $\mathrm{AUC}_{0-\infty}$ were significantly lower. Metformin is usually recommended in meals to improve gastrointestinal tolerance. At the same time, food does not affect the PK of sitagliptin. Therefore, Sitagliptin Phosphate/metformin Hydrochloride Tablets are recommended for dietary administration.

In this study, the parameters such as recoveries, matrix effects, linear range, lower limit of quantification, stability by specificity, precision, and accuracy specifications, were investigated to confirm the method of LC-MS/MS. Concentration of sitagliptin and metformin in human plasma showed good linear relationship within $1-500 \mathrm{ng} / \mathrm{mL}$ and $3-1500 \mathrm{ng} / \mathrm{mL}$. The relative standard deviation (RSD) values of intra- and inter-day precision were both less than $10 \%$. As an open-label study, AEs assessment may not be objective enough. When the test formulation passes the evaluation and enters the market, the efficacy and side effects need to be further explored. In addition, as this study is designed as a bioequivalence trial, The pharmacokinetic parameters and characteristics were all healthy adults. Further studies are needed to obtain pharmacokinetic parameters and make a recommendation about the medication with the Sitagliptin Phosphate/metformin Hydrochloride Tablets in special populations.

\section{Conclusion}

Our study evaluated the pharmacokinetics and bioequivalence of two Sitagliptin Phosphate/metformin Hydrochloride Tablets formulations via the pharmacokinetic parameters of sitagliptin and metformin. Both products showed good tolerability with a similar safety profile. The $90 \% \mathrm{Cls}$ of the ratios of the GM of the test and reference formulations for $\mathrm{C}_{\text {max }}, \mathrm{AUC}_{0-\mathrm{t}}$ and $\mathrm{AUC}_{0-\infty}$ were all within the predefined bioequivalence criteria range of $80-125 \%$, showing that the test formulation was bioequivalent to the reference formulation by any of detection indicators.

\section{Declarations}

\section{Ethics approval and consent to participate}

This clinical study protocol was approved by the Medical Ethics Committee at the Affiliated Hospital of Qingdao University and the trial was registered with the Clinical Trial Registry (trial ID: NCT04877106).

\section{Consent for publication}

Not applicable

\section{Availability of data and material}

The datasets generated during and/or analysed during the current study are available from the corresponding author on reasonable request. 


\section{Conflicts of Interest}

The authors declare no conflicts of interest.

\section{Funding}

This work was supported by grants from the National Major Scientific and Technological Special Project for “Significant New Drugs Development" (2020ZX09201-018, 2017ZX09304-024).

\section{Author contribution}

All authors completed this study together.

Xin Liu and Ping Shi planned and drafted the paper, and contributed to data quality control, analysis, and interpretation. Ting Li and Feifei Sun recorded the adverse events and made statistics on the adverse events. Yan-ping Liu and Shu-qin Liu contributed to data collection.

Yu Cao provided methodological guidance and support with data interpretation.

All authors reviewed and revised the manuscript.

\section{Acknowledgments}

The authors are grateful to Tonghua Dongbao Pharmaceutical Co., Ltd., Shandong, China, for their supports.

\section{References}

1. David R. Whiting, Leonor Guariguata, Clara Weil, Jonathan Shaw. IDF Diabetes Atlas: Global estimates of the prevalence of diabetes for 2011 and 2030[J]. Diabetes Research and Clinical Practice,2011,94(3).

2. Dai Q, Wang Z. Investigation of diabetes status in China[J]. Guide of China Medicine,2011,9(13):206208.

3. Michael Stumvoll,Barry J Goldstein,Timon W van Haeften. Type 2 diabetes: principles of pathogenesis and therapy[J]. The Lancet,2005,365(9467).

4. Pfeiffer AFH, Klein HH: The treatment of type 2 diabetes. Dtsch Arztebl Int 2014; 111(5): 69-82

5. Turner RC, Cull CA, Frighi V, et al. Glycemic control with diet, sulfonylurea, metformin, or insulin in patients with type 2 diabetes mellitus: progressive requirement for multiple therapies (UKPDS 49). UK Prospective Diabetes Study (UKPDS) Group. JAMA 1999; 281: 2005-12

6. Dhillon Sohita. Sitagliptin: a review of its use in the management of type 2 diabetes mellitus.[J]. Drugs,2010,70(4).

7. Lesley J. Scott. Sitagliptin: A Review in Type 2 Diabetes[J]. Drugs,2017,77(2). 
8. Goldstein Barry J,Feinglos Mark N,Lunceford Jared K,Johnson Jeremy,Williams-Herman Debora E. Effect of initial combination therapy with sitagliptin, a dipeptidyl peptidase-4 inhibitor, and metformin on glycemic control in patients with type 2 diabetes. [J]. Diabetes care,2007,30(8).

9. Chwieduk CM. Sitagliptin/metformin fixed-dose combination: in patients with type 2 diabetes mellitus. Drugs. 2011 Feb 12;71(3):349-61.

10. General Assembly of the World Medical, A. World Medical Association Declaration of Helsinki: ethical principles for medical research involving human subjects. J Am Coll Dent. 2014;81(3):14-8.

11. Switula D. Principles of good clinical practice (GCP) in clinical research. Sci Eng Ethics. 2000;6(1):71-7.

12. Berhane Seyoum. Sitagliptin and metformin - novel combination therapy[J]. Expert Opinion on Pharmacotherapy,2011,12(4).

13. Williams-Herman, Johnson,Teng,Luo,Davies,Kaufman,Goldstein,Amatruda. Efficacy and safety of initial combination therapy with sitagliptin and metformin in patients with type 2 diabetes: a 54-week study[J]. Current Medical Research and Opinion,2009,25(3).

14. Elizabeth Sanchez-Rangel,Silvio E. Inzucchi. Metformin: clinical use in type 2 diabetes[J]. Diabetologia,2017,60(9).

15. Seyoum B. Sitagliptin and metformin--novel combination therapy. Expert Opin Pharmacother. 2011 Mar;12(4):641-6.

16. Yang W, Guan Y, Shentu Y, Li Z, Johnson-Levonas AO, Engel SS, Kaufman KD, Goldstein BJ, Alba M. The addition of sitagliptin to ongoing metformin therapy significantly improves glycemic control in Chinese patients with type 2 diabetes. J Diabetes. 2012 Sep;4(3):227-37.

17. Scheen AJ. Pharmacokinetic and pharmacodynamic evaluation of sitagliptin plus metformin. Expert Opin Drug Metab Toxicol. 2010 Oct;6(10):1265-76.

\section{Tables}

Table 1 Pharmacokinetic parameters of sitagliptin and metformin test and reference formulations in the fasting group $(n=23)$ 


\begin{tabular}{|c|c|c|c|}
\hline \multirow[t]{2}{*}{ Parameters } & \multicolumn{2}{|c|}{ Arithmetic mean \pm SD $(\% \mathrm{CV})$} & \multirow[t]{2}{*}{ GMR $(90 \% \mathrm{Cl})$} \\
\hline & Test & Reference & \\
\hline \multicolumn{4}{|l|}{ Sitagliptin } \\
\hline $\mathrm{C}_{\max }(\mathrm{ng} / \mathrm{mL})$ & $182.37 \pm 63.53(34.8)$ & $162.93 \pm 37.16(22.8)$ & $101.70 \otimes 120.62$ \\
\hline$A \cup C_{0-t}(h * n g / m L)$ & $1704.19 \pm 32.54 \rrbracket 19.5 \rrbracket$ & $1676.46 \pm 269.80 \rrbracket 16.1 \rrbracket$ & $99.81 \otimes 105.61$ \\
\hline $\mathrm{AUC}_{0-\infty}\left(\mathrm{h}{ }^{\star} \mathrm{ng} / \mathrm{mL}\right)$ & $1744.90 \pm 35.79 \rrbracket 19.2 \rrbracket$ & 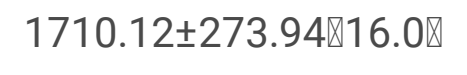 & $100.27 \rrbracket 106.12$ \\
\hline \multicolumn{4}{|l|}{ Metformin } \\
\hline $\mathrm{C}_{\max }(\mathrm{ng} / \mathrm{mL})$ & 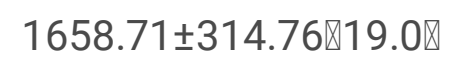 & $1666.23 \pm 330.44 \rrbracket 19.8 \rrbracket$ & $90.39 ه 111.48$ \\
\hline $\mathrm{AUC}_{0-\mathrm{t}}\left(\times 10^{4} \mathrm{~h}\right.$ ng/mL $)$ & $1.29 \pm 0.24 \llbracket 18.5 \rrbracket$ & $1.27 \pm 0.24 \rrbracket 19.0 \rrbracket$ & $94.76 \otimes 109.12$ \\
\hline $\mathrm{AUC}_{0-\infty}\left(\times 10^{4} \mathrm{~h} * \mathrm{ng} / \mathrm{mL}\right)$ & $1.32 \pm 0.25 \rrbracket 18.9 \rrbracket$ & $1.29 \pm 0.25 \rrbracket 19.1 \rrbracket$ & $95.76 \rrbracket 110.38$ \\
\hline
\end{tabular}

Table 2 Pharmacokinetic parameters of sitagliptin and metformin test and reference formulations in the fed group $(n=23)$

\begin{tabular}{|c|c|c|c|}
\hline \multirow[t]{2}{*}{ Parameters } & \multicolumn{2}{|l|}{ Arithmetic mean \pm SD $(\% C V)$} & \multirow[t]{2}{*}{ GMR $(90 \% \mathrm{Cl})$} \\
\hline & Test & Reference & \\
\hline \multicolumn{4}{|l|}{ Sitagliptin } \\
\hline$C_{\max }(n g / m L)$ & $201.379 \pm 39.667(19.7)$ & $181.746 \pm 33.439(18.4)$ & $102.12 \otimes 117.31$ \\
\hline $\mathrm{AUC}_{0-\mathrm{t}}(\mathrm{h} * \mathrm{ng} / \mathrm{mL})$ & $1727.80 \pm 258.28(14.9)$ & $1665.89 \pm 256.74(15.4)$ & $100.80 \otimes 107.81$ \\
\hline $\mathrm{AUC}_{0-\infty}(\mathrm{h} * \mathrm{ng} / \mathrm{mL})$ & $1762.92 \pm 257.47(14.6)$ & $1699.38 \pm 254.27(15.0)$ & $100.82 \bigotimes 107.78$ \\
\hline \multicolumn{4}{|l|}{ Metformin } \\
\hline $\mathrm{C}_{\max }(\mathrm{ng} / \mathrm{mL})$ & $1270.960 \pm 205.903(16.2) \rrbracket$ & $1261.443 \pm 184.167(14.6)$ & $95.53 \rrbracket 105.22$ \\
\hline $\mathrm{AUC}_{0-\mathrm{t}}\left(\times 10^{4} \mathrm{~h}^{\star} \mathrm{ng} / \mathrm{mL}\right)$ & $1.14 \pm 0.20(17.7)$ & $1.16 \pm 0.19(16.3)$ & $92.76 ه 103.07$ \\
\hline $\mathrm{AUC}_{0-\infty}\left(\times 10^{4} \mathrm{~h}^{\star} \mathrm{ng} / \mathrm{mL}\right)$ & $1.17 \pm 0.22(18.5)$ & $1.19 \pm 0.19(16.1)$ & $93.40 \otimes 104.14$ \\
\hline
\end{tabular}

Table 3 Adverse events in the fasting group 


\begin{tabular}{|llll|}
\hline Random number & AEs & Treatment & Relationship with the drugs \\
\hline K009 & Nodal tachycardia & R & Probably related \\
\hline K016 & Nausea & T & May be irrelevant \\
\hline K017 & Hyperuricemia & R & Probably related \\
\hline K020 & Nausea & Before treatment & Definitely not \\
\hline K024 & Dizziness & Before treatment & Definitely not \\
\hline
\end{tabular}

Table 4 Adverse events in the fed group

\begin{tabular}{|llll|}
\hline Random number & AEs & Treatment & Relationship with the drugs \\
\hline C001 & Hyperpotassemia & R & Probably related \\
\hline C004 & Nodal tachycardia & R & Definitely not \\
\hline C016 & Urinary leukocytosis & R & Probably related \\
\hline C023 & Borderline premature beats & R & Probably related \\
\hline
\end{tabular}

\section{Figures}




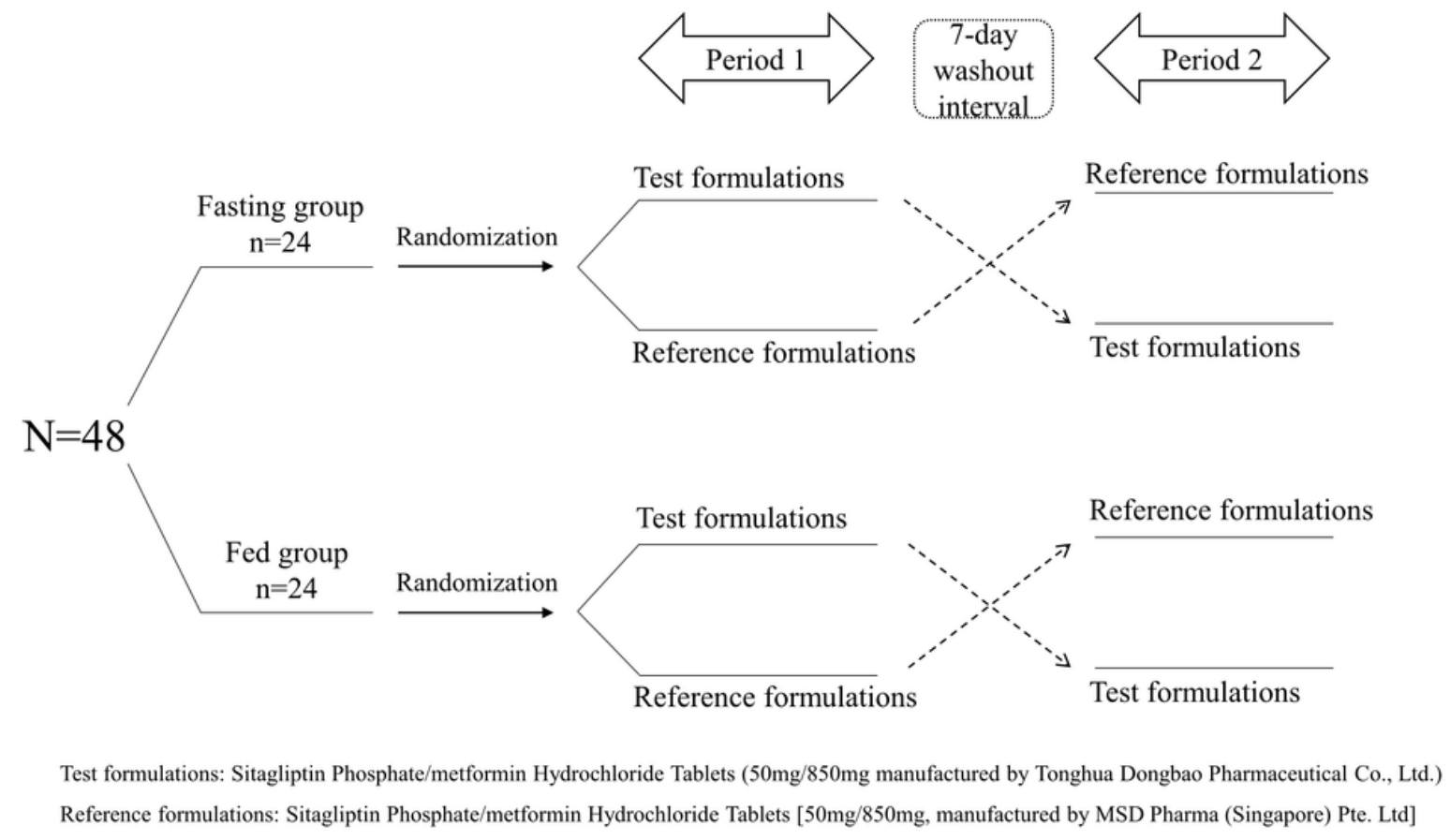

\section{Figure 1}

Sitagliptin Phosphate/metformin Hydrochloride Tablets [50mg/850mg, batch no. M047893, manufactured by MSD Pharma (Singapore) Pte.Ltd] as reference formulations and Sitagliptin Phosphate/metformin Hydrochloride Tablets (50mg/850mg, batch no. 161006, manufactured by Tonghua Dongbao Pharmaceutical Co., Ltd.) as test formulations were used in this study. All study drugs were provided by the sponsor (Tonghua Dongbao Pharmaceutical Co., Ltd.) at no cost. All subjects were hospitalized in the phase I clinical research center on the day before dosing and placed on a uniform diet during hospitalization. Subjects in the fasting group ( 24 subjects) were asked to fast overnight $(10 \mathrm{~h}$ ) before administration, while the subjects in the fed group (24 subjects) needed to take an additional highfat, high-calorie standard meal $30 \mathrm{~min}$ prior to the administration. Test and reference formulations of Sitagliptin Phosphate/metformin Hydrochloride Tablets was swallowed with $240 \mathrm{ml}$ water at room temperature. Subjects were allocated to one of two groups randomly and equally with a 7-day washout interval between the two periods 

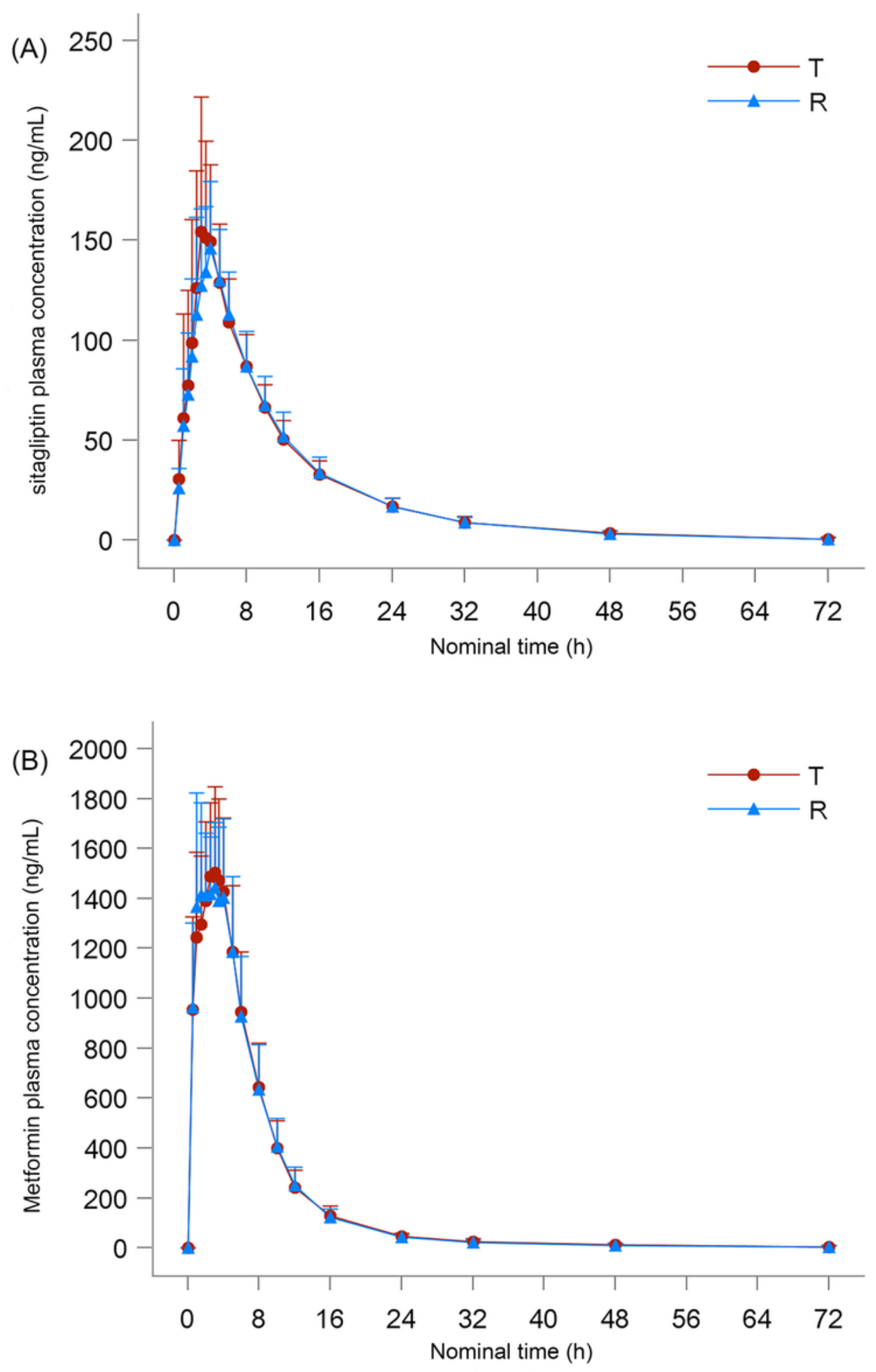

Figure 2

The plasma concentration-time profiles for sitagliptin and metformin under fasting conditions are shown respectively 

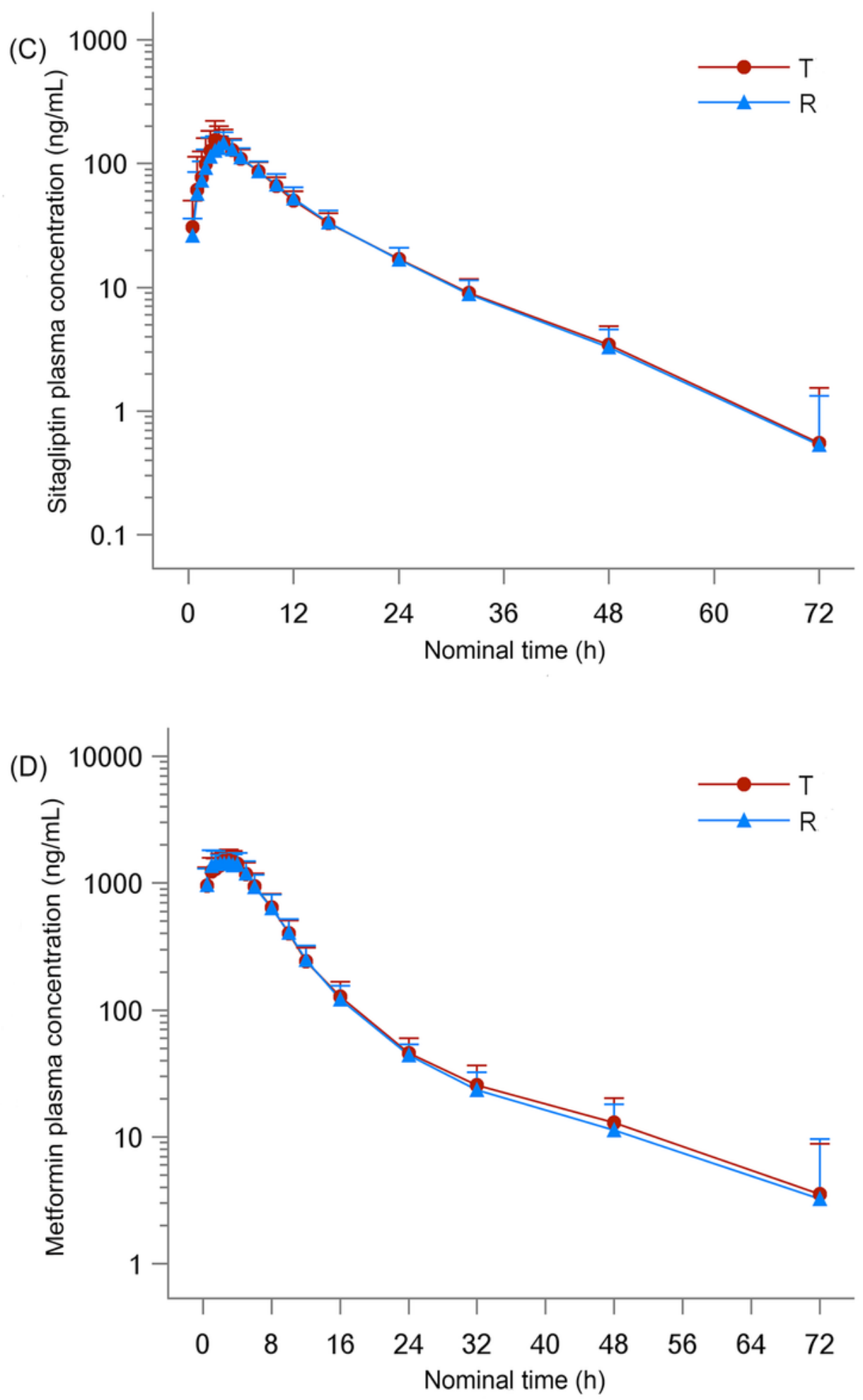

Figure 3

The plasma concentration-time profiles for sitagliptin and metformin under fasting conditions are shown respectively 

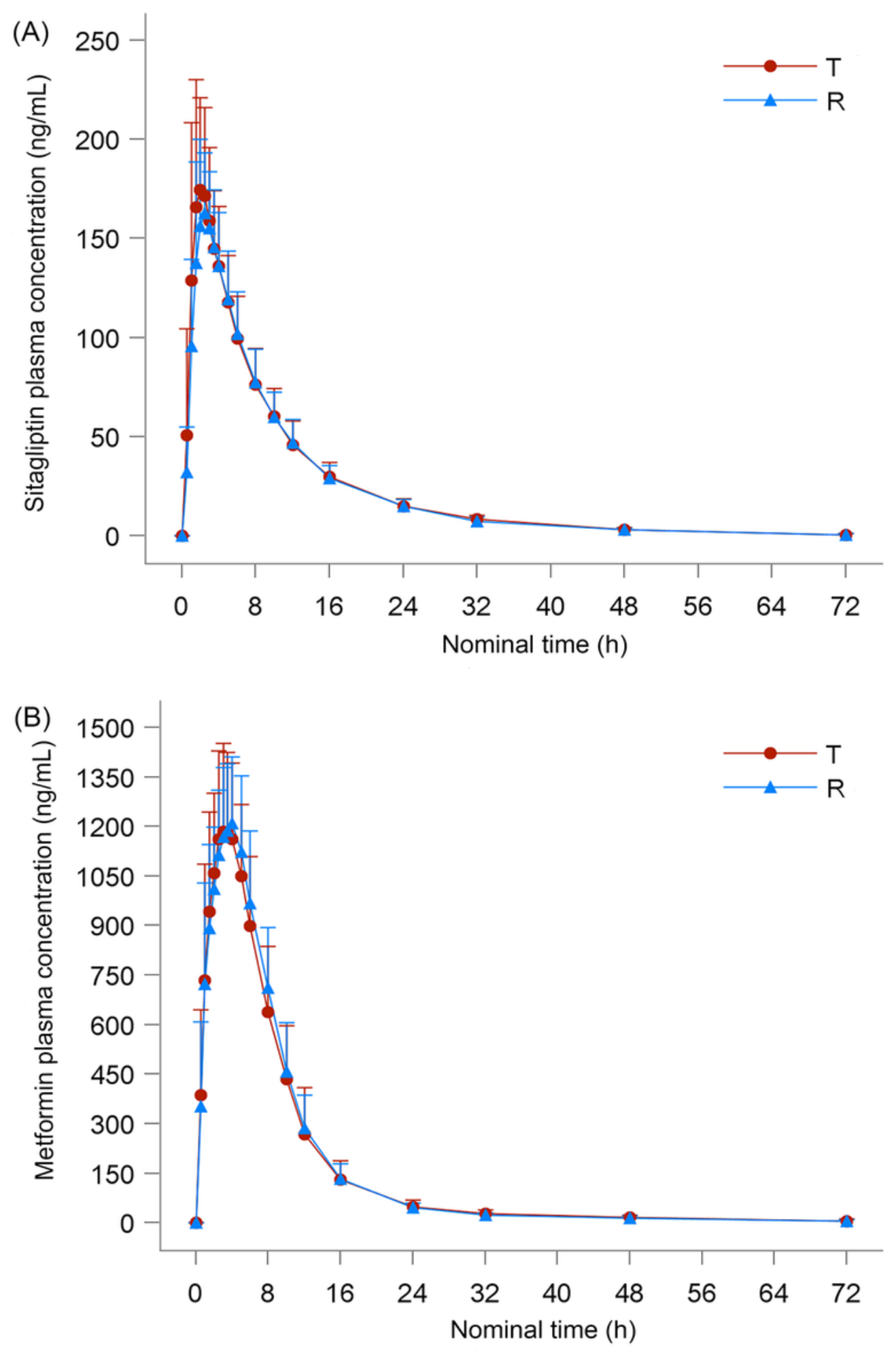

Figure 4

The mean plasma concentration-time profiles for sitagliptin and metformin under fed conditions are shown respectively 

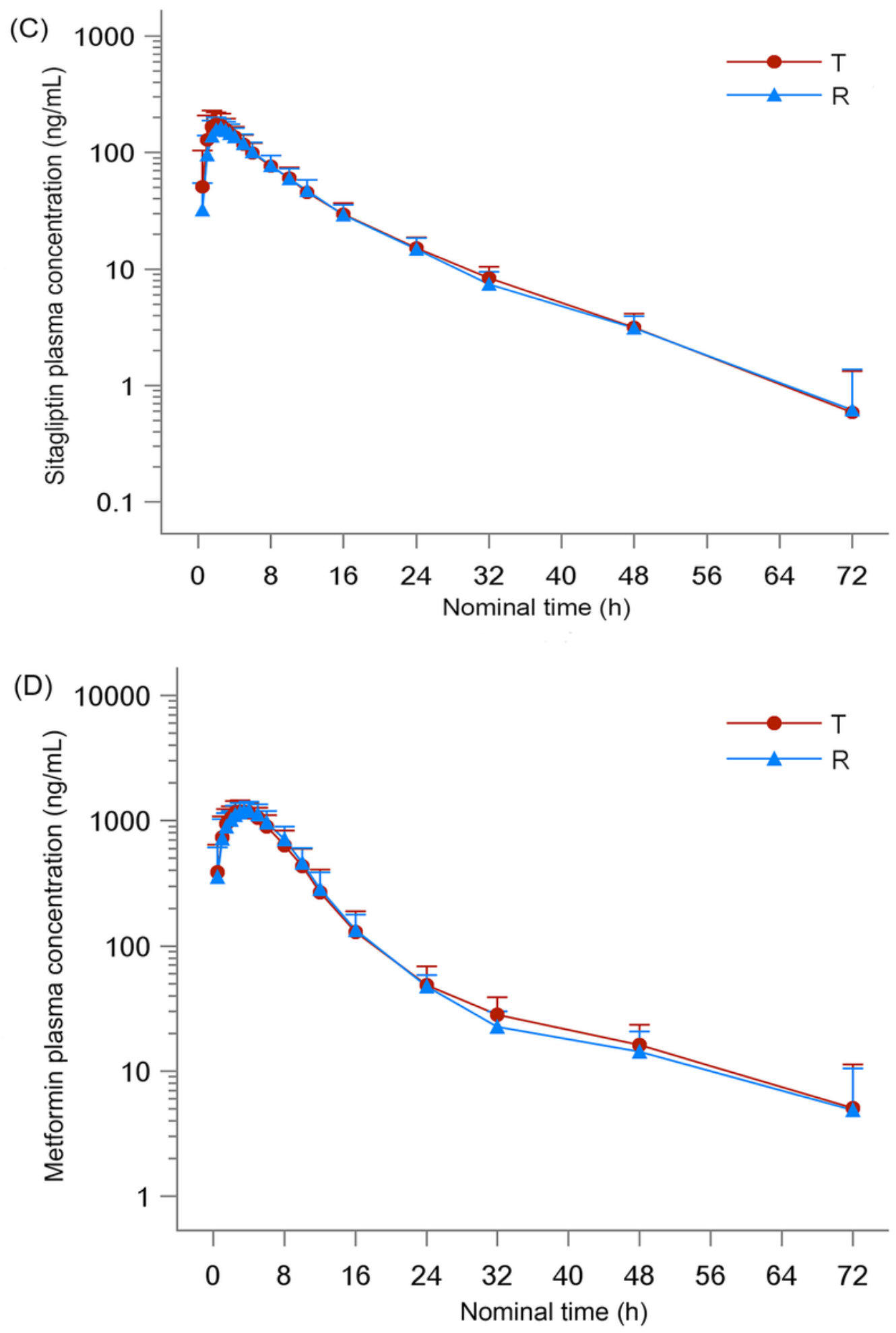

Figure 5

The mean plasma concentration-time profiles for sitagliptin and metformin under fed conditions are shown respectively 